\title{
EVOLUÇÃO DO CRESCIMENTO E DESENVOLVIMENTO NEUROPSICOMOTOR DE CRIANÇAS PRÉ-ESCOLARES DE ZERO A DOIS ANOS DO MUNICÍPIO DE GOIÂNIA (GO)
}

\author{
EVOLUTION OF GROWTH AND DEVELOPMENT OF \\ CHILDREN PRESCHOOLERS ZERO-TWO YEARS IN \\ THE CITY OF GOIÂNIA (GO)
}

Ana Karolina Paiva Braga ${ }^{1}$

Juliana Campos Rodovalho ${ }^{1}$

Cibelle Kayenne Martins Roberto Formiga ${ }^{2}$

Braga AKP; Rodovalho JC; Formiga CKMR. Evolução do crescimento e desenvolvimento neuropsicomotor de crianças pré-escolares de zero a dois anos do município de Goiânia (GO). Rev Bras Cresc e Desenv Hum 2011; 21(2): 230-239.

\section{RESUMO}

Os objetivos do estudo foram analisar a evolução do crescimento e desenvolvimento de crianças pré-escolares em creches de Goiânia e verificar a associação entre fatores de risco no crescimento e desenvolvimento das crianças. Participaram 39 crianças, de ambos os sexos, de zero a dois anos de idade. As crianças foram avaliadas em três momentos, com intervalo de dois meses entre as avaliações. O crescimento foi avaliado pelas medidas antropométricas de peso e altura e o desenvolvimento foi avaliado pelo Teste de Denver II. A maioria das crianças apresentou crescimento normal. Na primeira avaliação $46 \%$ das crianças apresentaram risco para o desenvolvimento, na segunda avaliação $28 \%$ de risco e na última avaliação $44 \%$, sendo a linguagem a área mais defasada. Variáveis que tiveram relação com o déficit do crescimento e atraso no desenvolvimento: maior idade materna, menor Apgar $5^{\circ}$ minuto, crianças do sexo masculino, menor peso adquirido na gravidez e mães desempregadas.

Palavras-chave: crescimento infantil; desenvolvimento infantil; pré-escolar; fatores de risco; deteç̧ão de atrasos.

Fisioterapeutas pela Universidade Estadual de Goiás (UEG).

2 Fisioterapeuta, Doutora em Ciências Médicas pela FMRP/USP, Professora Adjunta da Universidade Estadual de Goiás (UEG). Correspondência para: Profa. Dra. Cibelle Kayenne Martins Roberto Formiga - Rua: Mamoré, Quadra F6, Lote 12 Residencial Araguaia - Alphaville Flamboyant, Goiânia - GO, CEP 74883-015. Telefone: (62) 3246-0452. Celular: (62) 8403-3167 E-mail: cibellekayenne@gmail.com 


\begin{abstract}
The study aimed to analyze growth and development evolution of preschool children in daycare centers from Goiânia, check the association between risk factors in children growth and development. We evaluated 39 children of both sexes, 0-2 years old. Children were assessed on three different occasions with an interval of two months between assessments. Growth was assessed by anthropometric measurements of height and weight and development was assessed by the Denver Test II. Most children had normal growth. In the first assessment $46 \%$ of children were at risk for the development, $28 \%$ in the second evaluation and risk assessment in the last $44 \%$ of the language area later. The following variables were associated with growth deficits and delayed development: increased maternal age, lower Apgar score at 5 minutes, male, less weight gained during pregnancy and unemployed mothers.
\end{abstract}

Key words: child growth, child development, preschool, risk factors, detection of delay.

\section{INTRODUÇÃO}

A saúde da criança no Brasil estruturase, prioritariamente, em torno da assistência em puericultura, com enfoque no controle do crescimento e desenvolvimento infantil ${ }^{1}$. O conhecimento da situação de crescimento e desenvolvimento de crianças cuidadas em creches, permite à implementação de ações de promoção e prevenção da saúde, contribuindo para o delineamento de políticas públicas, articulação intersetorial e conscientização da população, proporcionando à criança atingir um desenvolvimento sadio e harmonioso ${ }^{2,3,4}$.

De 1996 a 2006 o número de crianças de 0 a 3 anos de idade que frequentam creches passou de $7,4 \%$ para $15,5 \%$, devido às transformações socioeconômicas da população brasileira $^{5,6}$. Creches são instituições que atendem crianças na idade pré-escolar ( 0 - 6 anos de idade), uma fase de aquisição e aperfeiçoamento das habilidades motoras, adquirindo os modelos de coordenação essenciais para posteriores performances habilidosas ${ }^{7,8}$.

Crianças que frequentam centros infantis têm maior risco de adquirir infecções, acarretando maior probabilidade para atraso no desenvolvimento e crescimento infanti1 ${ }^{9,10}$. Nos países em desenvolvimento, grande parte da população vive em estado de pobreza ou miséria, o que pode interferir na qualidade das refeições, onde as crianças recebem os elementos construtivos necessários ao seu crescimento ${ }^{11}$.

Acompanhar a criança nos dois primeiros anos de vida extra-uterina é de fundamental importância, pois é nesta etapa que o organismo mais cresce e amadurece, estando mais sujeito aos agravos.

$\mathrm{O}$ crescimento se refere às mudanças de ordem quantitativa, no aumento da estatura e do peso corporal ${ }^{12}$. O desenvolvimento, por sua vez, pode ser definido como a capacidade do indivíduo de realizar funções cada vez mais complexas ${ }^{13}$. O crescimento e o desenvolvimento sofrem a influência de fatores intrínse$\cos$ (geneticamente determinados) e extrínsecos (condições socioeconômicas, de saúde e nutrição, assim como o ambiente em que a criança vive após o nascimento) ${ }^{14}$.

Todo ser humano nasce com potencial genético de crescimento e desenvolvimento, que poderá ou não ser alcançado, dependendo das condições de vida que lhe sejam propiciadas ${ }^{3}$.

Existe o risco de 200 milhões de crianças abaixo de cinco anos em países em desenvolvimento não alcançarem seu potencial de desenvolvimento cognitivo devido à pobreza, 
saúde e nutrição deficitárias e falta de cuidados e estimulação adequados ${ }^{15}$.

No trabalho de Pilz \& Schermann ${ }^{16}$ realizado em Canoas (RS), com 197 crianças de 0 a 6 anos, $27 \%$ destas apresentaram teste de triagem de Denver II suspeito de atraso no desenvolvimento. A baixa renda familiar foi o fator que demonstrou ter maior associação com a suspeita de atraso.

Diante da diversidade de fatores de risco envolvidos no processo de crescimento e desenvolvimento, os objetivos são os de analisar a evolução da taxa de crescimento e desenvolvimento de crianças pré-escolares em creches municipais de Goiânia e verificar a associação entre fatores de risco de natureza biológica e ambiental no crescimento e desenvolvimento das crianças pré-escolares.

\section{MÉTODO}

Estudo de delineamento longitudinal, em que participaram 39 crianças, sendo 26 (67\%) do sexo feminino e 13 (33\%) do sexo masculino, matriculadas em cinco CMEIs (Centros Municipais de Educação Infantil) da cidade de Goiânia (Goiás).

Os critérios para exclusão foram: prematuridade, necessidade de tratamento intensivo no pós-parto imediato e evidência de infecções congênitas, anomalias cromossômicas e malformações, desistência ou a não autorização dos responsáveis.

As crianças foram avaliadas em três momentos, com intervalos de aproximadamente dois meses entre as avaliações. Na primeira avaliação, a faixa etária variou de 5,2 a 23,5 meses de idade. Na segunda avaliação a idade foi de 7,4 a 26,5 meses. E na terceira avaliação a idade variou de 10,5 a 29 meses.

O estudo foi aprovado pelo Comitê de Ética em Pesquisa da Universidade Federal de Goiás e pela Secretaria Municipal de Educação de Goiás. Os pais que concordaram com a participação de seus filhos na pesquisa assinaram o Termo de Consentimento Livre e Esclarecido (TCLE).

Foram coletados os dados das crianças com informações referentes à gestação, ao parto e dados neonatais, bem como o peso e o comprimento ao nascimento, Questionário SócioEconômico e Formulário da ABEP (Associação Brasileira de Empresa de Pesquisa $)^{17}$.

Para avaliar o crescimento dos lactentes foram utilizados: Fita métrica para avaliação do perímetro cefálico e perímetro torácico; Antropômetro horizontal para medição do comprimento; Balança digital para avaliação do peso; e as curvas de Peso/Idade, Estatura/Idade, Peso/Estatura, Perímetro cefálico e Perímetro torácico adotadas pela Organização Mundial da Saúde (OMS) como padrão internacional ${ }^{14}$.

Para avaliar o desenvolvimento neuropsicomotor foram utilizados: Teste de Triagem de Denver II adaptado para o português, composto por 125 itens que são divididos em quatro domínios: a) Pessoal-Social; b) Linguagem; c) Motricidade Fina Adaptativa e d) Motricidade Ampla. Para classificação da criança quanto ao desenvolvimento foram considerados casos indicativos de suspeita de atraso no desenvolvimento aqueles em que a criança apresentou dois ou mais itens de "cuidados", isto é, a criança falha na realização da tarefa quando $75-90 \%$ das crianças da faixa etária o realiza, e/ou um ou mais itens de "atrasos", ou seja, não realização do item quando $90 \%$ ou mais das crianças o realiza ${ }^{18}$; Máquina fotográfica para ilustração das tarefas do teste.

Após coleta dos dados foi realizada a preparação do Banco de Dados. Comparou-se a evolução do crescimento e do desenvolvimento entre os resultados das três avaliações usando o Teste de Análise de Variância com grupos dependentes e o Teste de Friedman, respectivamente. Por último, foram realizadas correlações entre as variáveis biológicas, ambientais e os resultados das avaliações do crescimento e desenvolvimento através do Tes- 
te de Correlação de Spearman. Todas as análises foram realizadas utilizando-se o programa estatístico SPSS (Statistical Package for the Social Sciences) e foi considerado o nível de significância estatística de 5\% ( p d" 0,05).

\section{RESULTADOS}

Os resultados da classificação do Peso, Comprimento e relação Peso/Estatura são representados na figura 1 .

Tabela 1: Classificação das crianças quanto ao Peso, Comprimento e Relação Peso/Estatura nas três avaliações.

\begin{tabular}{|c|c|c|c|c|}
\hline & Classificação & $1^{a}$ Avaliação & $2^{a}$ Avaliação & $3^{\text {a }}$ Avaliação \\
\hline \multirow[t]{4}{*}{ Peso } & Normal & $81 \%$ & $86 \%$ & $79 \%$ \\
\hline & Risco & $8 \%$ & $3 \%$ & $3 \%$ \\
\hline & Sobrepeso & $3 \%$ & $3 \%$ & $3 \%$ \\
\hline & Peso baixo & $8 \%$ & $8 \%$ & $15 \%$ \\
\hline \multirow[t]{3}{*}{ Comprimento } & Normal (P entre 3 - 97) & $97 \%$ & $97 \%$ & $97 \%$ \\
\hline & Abaixo do Normal & $0 \%$ & $3 \%$ & $3 \%$ \\
\hline & Acima do Normal & $3 \%$ & $0 \%$ & $0 \%$ \\
\hline Relação & Normal & $94 \%$ & $92 \%$ & $90 \%$ \\
\hline \multirow[t]{2}{*}{ Peso/Estatura } & Desnutrição & $3 \%$ & $3 \%$ & $3 \%$ \\
\hline & Sobrepeso & $3 \%$ & $5 \%$ & $7 \%$ \\
\hline
\end{tabular}

Nas três avaliações a maioria das crianças apresentou peso dentro da faixa de normalidade $(81 \%, 86 \%$ e $79 \%$, respectivamente), variando de uma avaliação para outra nas porcentagens de risco nutricional e sobrepeso. Quanto ao comprimento, a maioria das crianças (97\%) mostrou-se com o comprimento dentro da faixa de normalidade nas três avaliações. Na relação Peso/Esta-

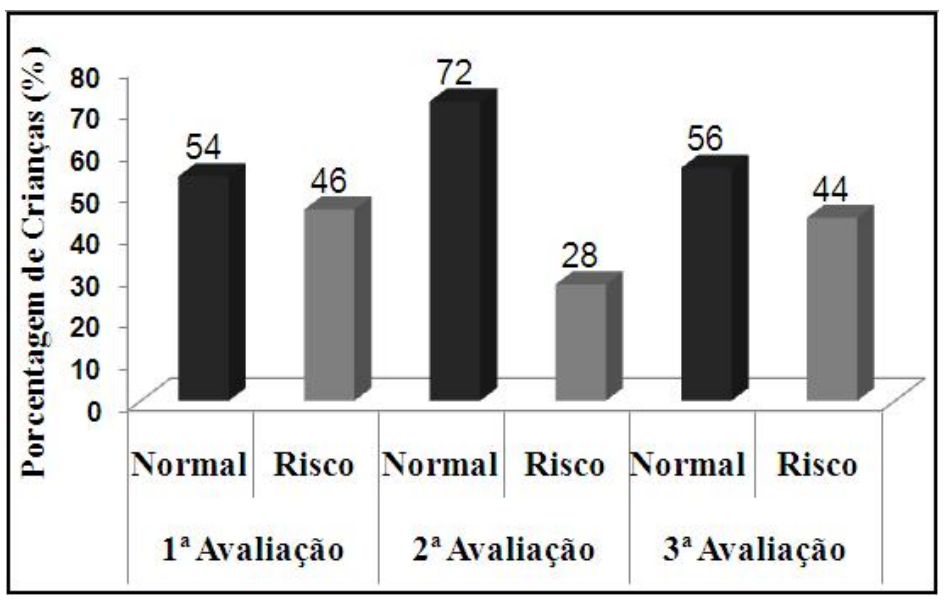

Figura 1: Classificação do desenvolvimento neuropsicomotor Global segundo o teste de Denver II. * Teste de Friedman: $\mathrm{p}=0,057$. 
tura a maior parte das crianças foi classificada dentro da faixa de normalidade nas três avaliações $(94 \%, 92 \%$ e $90 \%$ respectivamente).
A figura 2 apresenta o resultado final do desenvolvimento do Teste de Denver II, nas três avalições realizadas.

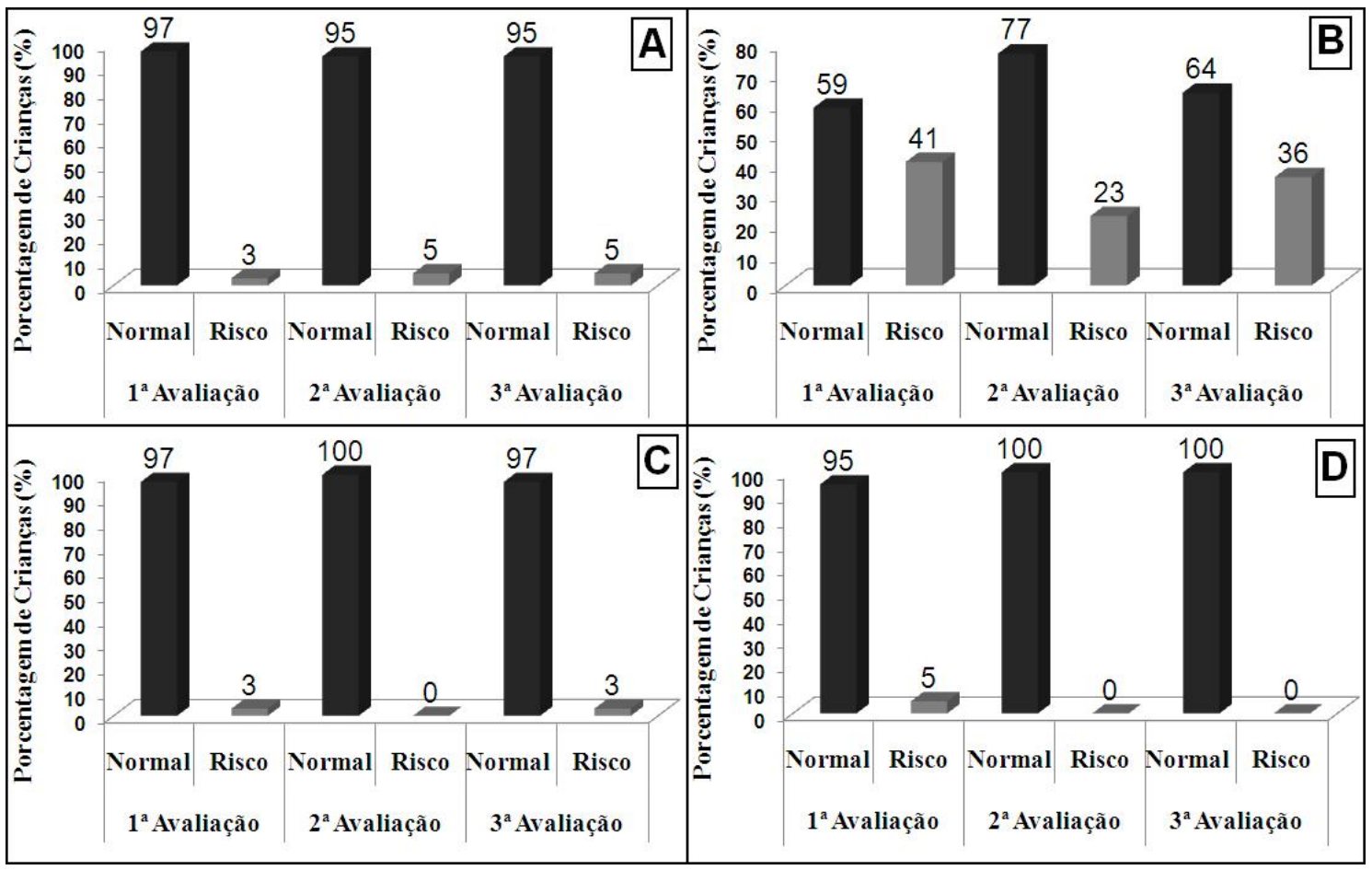

Figura 2: Classificação do desenvolvimento neuropsicomotor segundo o teste de Denver II: Área Pessoal Social (A), Área da Linguagem (B), Área Motor fino (C) e Área Motor amplo (D). * Teste de Friedman: Área Pessoal social $(\mathrm{p}=0,779)$, Área Motor fino $(\mathrm{p}=0,607)$, Área da Linguagem $\left(\mathrm{p}=0,05^{*}\right)$, Área Motor amplo $(\mathrm{p}=0,135)$.

Na primeira avaliação $46 \%$ das crianças apresentaram risco para problemas de desenvolvimento, na segunda avaliação $28 \%$ de risco e na última avaliação $44 \%$.

A figura 3 apresenta os resultados do desenvolvimento do Teste de Denver II, nas três avalições realizadas, segundo as áreas avaliadas.

A distribuição das crianças de acordo com os resultados do Teste de Denver II, segundo áreas do desenvolvimento apontou a linguagem como a área mais comprometida, com risco de atraso no desenvolvimento de $41 \%, 23 \%$ e $36 \%$, nas três avaliações.
As variáveis que mostraram associação significativa com suspeita de atraso no crescimento encontram-se na tabela 2.

Quanto maior a idade da mãe, maior o risco de atraso para o comprimento da criança; crianças com menor Apgar no $5^{\circ}$ minito apresentaram menor PT durante as avaliações; o sexo masculino esteve relacionado com menor peso; quanto maior o peso adquirido na gravidez, maior o peso da criança na $2^{\mathrm{a}}$ avaliação.

Verificou-se que quanto mais baixa a classificação para peso, comprimento, PC e PT, maior o risco de atraso do desenvolvimento, 
Tabela 2: Associação entre crescimento, variáveis biológicas e variáveis ambientais

\begin{tabular}{|c|c|c|c|}
\hline Variável 1 & Variável 2 & $\begin{array}{l}\text { Coef. Correlação } \\
\text { (r) }\end{array}$ & $\begin{array}{c}\text { Valor de } \\
p^{*}\end{array}$ \\
\hline PT da $1^{\mathrm{a}} \mathrm{AV}$ & Apgar $5^{\circ}$ minuto & 0,349 & 0,034 \\
\hline $\begin{array}{l}\text { Classificação do peso } \\
\text { da } 2^{\mathrm{a}} \mathrm{AV}\end{array}$ & $\begin{array}{l}\text { Sexo da criança } \\
\text { Peso adquirido na } \\
\text { gravidez }\end{array}$ & $\begin{array}{c}-0,354 \\
0,347\end{array}$ & $\begin{array}{l}0,027 \\
0,033\end{array}$ \\
\hline $\begin{array}{l}\text { Classificação do peso } \\
\text { da } 3^{\mathrm{a}} \mathrm{AV}\end{array}$ & Sexo da criança & $-0,406$ & 0,010 \\
\hline $\begin{array}{l}\text { Comprimento da } \\
3^{\mathrm{a}} \mathrm{AV}\end{array}$ & Idade da mãe & $-0,318$ & 0,048 \\
\hline
\end{tabular}

* Teste de Correlação de Spearman. PT: Perímetro torácico; AV: Avaliação

seja global ou separadamente segundo a distribuição em áreas do desenvolvimento, principalmente para a linguagem.
As variáveis que apresentam associação significativa com suspeita de atraso no desenvolvimento encontram-se na tabela 3 .

Tabela 3: Associação entre desenvolvimento, variáveis biológicas e variáveis ambientais.

\begin{tabular}{|c|c|c|c|}
\hline Variável 1 & Variável 2 & $\begin{array}{c}\text { Coef. Correlação } \\
\text { (r) }\end{array}$ & $\begin{array}{c}\text { Valor de } \\
\mathbf{p}^{*}\end{array}$ \\
\hline \multirow[t]{5}{*}{ Linguagem da $1^{\mathrm{a}} \mathrm{AV}$} & Idade da mãe & 0,332 & 0,039 \\
\hline & Global da $1^{\mathrm{a}} \mathrm{AV}$ & 0,901 & $<0,001$ \\
\hline & Linguagem da $2^{\mathrm{a}} \mathrm{AV}$ & 0,533 & $<0,001$ \\
\hline & Global da $2^{\mathrm{a}} \mathrm{AV}$ & 0,636 & $<0,001$ \\
\hline & Linguagem da $3^{\mathrm{a}} \mathrm{AV}$ & 0,463 & 0,003 \\
\hline \multirow[t]{5}{*}{ Motor grosso da $1^{\mathrm{a}} \mathrm{AV}$} & Global da $3^{\mathrm{a}} \mathrm{AV}$ & 0,423 & 0,007 \\
\hline & Linguagem da $2^{\mathrm{a}} \mathrm{AV}$ & 0,424 & 0,007 \\
\hline & Global da $2^{\mathrm{a}} \mathrm{AV}$ & 0,371 & 0,020 \\
\hline & Pessoal social da $3^{\mathrm{a}} \mathrm{AV}$ & 0,473 & 0,002 \\
\hline & Motor fino da $3^{\mathrm{a}} \mathrm{AV}$ & 0,698 & $<0,001$ \\
\hline \multirow[t]{2}{*}{$\overline{\text { Global da } 1^{\mathrm{a}} \mathrm{AV}}$} & Global da $2^{\mathrm{a}} \mathrm{AV}$ & 0,563 & $<0,001$ \\
\hline & Global da $3^{\mathrm{a}} \mathrm{AV}$ & 0,327 & 0,042 \\
\hline \multirow[t]{2}{*}{ Pessoal social da $2^{\mathrm{a}} \mathrm{AV}$} & Sexo da criança & 0,329 & 0,041 \\
\hline & Global da $2^{\mathrm{a}} \mathrm{AV}$ & 0,371 & 0,020 \\
\hline Pessoal social da $3^{\mathrm{a}} \mathrm{AV}$ & Motor fino da $3^{\mathrm{a}} \mathrm{AV}$ & 0,698 & $<0,001$ \\
\hline$\overline{\text { Linguagem da } 3^{\mathrm{a}} \mathrm{AV}}$ & Apgar 5 & $-0,433$ & 0,007 \\
\hline$\overline{\text { Motor fino da } 3^{\mathrm{a}} \mathrm{AV}}$ & Se a mãe está empregada & $-0,480$ & 0,002 \\
\hline Global da $3^{\mathrm{a}}$ avaliação & Apgar 5 & $-0,418$ & 0,010 \\
\hline
\end{tabular}

* Teste de Correlação de Spearman.

PT: Perímetro torácico; AV: Avaliação 
Quanto maior a idade da mãe, maior o risco de atraso para o comprimento e o desenvolvimento da linguagem; crianças com menor apgar apresentaram maior risco de atraso para o desenvolvimento da linguagem e do global da $3^{\text {a }}$ avaliação; o sexo masculino esteve relacionado com maior atraso no desenvolvimento pessoal social; mães desempregadas apresentaram maior atraso no desenvolvimento da área motor fino da $3^{\mathrm{a}}$ avaliação; o atraso na área da linguagem relacionou-se com o atraso global do desenvolvimento.

\section{DISCUSSÃO}

A avaliação do estado nutricional das crianças detectou prevalência de $8 \%$ de risco nutricional e $8 \%$ de sobrepeso inicialmente, e posteriormente com $3 \%$ de risco nutricional e $15 \%$ de sobrepeso para Peso/Idade; inicialmente $3 \%$ das crianças estavam com alta estatura, porém ao final das avaliações 3\% estavam com baixa estatura; em relação ao Peso/Estatura, na primeira avaliação $3 \%$ das crianças apresentaram desnutrição e $3 \%$ sobrepeso, na segunda $3 \%$ apresentaram desnutrição e 5\% sobrepeso e na última avaliação 3\% de desnutrição e 7\% sobrepeso. Os valores de sobrepeso apresentaram-se abaixo do percentual encontrado em certo estudo ${ }^{19}$, que avaliou crianças de 2 a 6 anos de idade de uma creche de Duque de Caxias e encontrou $27 \%$ de sobrepeso. Em relação ao comprimento, este estudo diferiu do presente, visto que apresentou uma prevalência de risco de baixa estatura de $28 \%$.

Outro estudo ${ }^{20}$ que encontrou alta prevalência de obesidade $(34,4 \%)$ avaliou o crescimento de crianças entre 2 e 6 anos de idade matriculadas em escolas privadas no município de São Paulo e detectou como fator de proteção contra sobrepeso o aleitamento materno exclusivo por 6 meses ou mais. No presente estudo, o sobrepeso foi menor e o tempo mé- dio de aleitamento materno exclusivo foi de 4 meses, com o máximo em 7 meses.

Estudo $^{21}$ que avaliou o crescimento de crianças de 6 a 60 meses de idade, atendidas em creches, encontrou dados semelhantes ao do presente estudo, com a prevalência de desnutrição, segundo o índice Peso/idade e Peso/ estatura, de $5,1 \%$ e $4,5 \%$ respectivamente, estando a baixa estatura em 3,8\% das crianças. $\mathrm{O}$ déficit nutricional do estudo foi associado com peso ao nascer, escolaridade paterna e renda per capita, que diferiu do presente estudo, onde o déficit esteve associado à maior idade materna, menor Apgar no $5^{\circ}$ minuto, crianças do sexo masculino e menor peso adquirido na gravidez pela mãe. As associações com o crescimento encontradas no presente estudo revelam a importância de um maior acompanhamento nutricional para as crianças do sexo masculino de mães mais velhas, que apresentaram menor Apgar no $5^{\circ}$ minuto, e também um maior acompanhamento nutricional para as mães durante a gestação.

Uma pesquisa ${ }^{22}$, encontrou que os lactentes de mães adolescentes apresentaram um pior desempenho de crescimento, quando comparados com o dos filhos de mães adultas. Este dado não corrobora com os do presente estudo, que observou que quanto maior a idade da mãe, maior foi o risco encontrado para atraso no comprimento das crianças. Vale lembrar que a média de idade das mães do presente estudo foi de 28 anos, não havendo mãe adolescente.

No que diz respeito ao tipo de estudo longitudinal, é importante destacar que não foram encontrados estudos semelhantes ao realizado, uma vez que todos os estudos que avaliam o crescimento de pré-escolares são de delineamento transversal e no presente estudo o objetivo era verificar como o crescimento e desenvolvimento dessas crianças se comportava ao longo do tempo.

Quanto aos resultados do desenvolvimento, verificou-se que $46 \%$ das crianças apre- 
sentaram risco para problemas de desenvolvimento na primeira avaliação, $28 \%$ de risco na segunda avaliação e $44 \%$ de risco na última avaliação. O presente estudo corrobora com certo estudo ${ }^{23}$ nas duas primeiras avaliações, que comparou o desenvolvimento neuropsicomotor na idade de 12 meses de crianças nascidas na cidade de Pelotas-Brasil, onde a prevalência de suspeita de atraso de desenvolvimento caiu de 37,1\% em 1993 para 21,4\% em 2004.

Ao analisar a área do desenvolvimento mais comprometida durante as avaliações, observa-se que a linguagem foi a área com maior risco. Uma possível explicação para este resultado estaria nos fatores sociais relacionados à estimulação para que os padrões linguísticos se desenvolvam. Outras prováveis explicações para tal resultado encontram-se no fato de que as crianças permaneciam por tempo integral nos CMEIs, com pouco contato com os pais, e o grande contingente de crianças que está sob responsabilidade de cada cuidadora na creche pode ter dificultado a estimulação da linguagem, frente às necessidades básicas de cuidados de rotina do ambiente escolar (banho, troca e alimentação).

Tais resultados foram semelhantes a alguns estudos ${ }^{3,5}$, que avaliaram o desenvolvimento neuropsicomotor pelo Teste de Denver II em crianças frequentadoras de creches, e detectou a linguagem como a área mais acometida.

Certo estudo ${ }^{24}$ ao avaliar a aquisição da linguagem através do Teste de Denver II, observou que a suspeita de atraso para o desenvolvimento foi maior nas crianças do sexo masculino (58,5\%). O sexo masculino apresentou-se no presente estudo com maior atraso no desenvolvimento pessoal social. Outros estudos $^{16,25}$ também encontraram relação entre 0 sexo masculino e maior suspeita de atraso no desenvolvimento neuropsicomotor em comparação ao sexo feminino. Determinado estudo ${ }^{26}$, que avaliou o desenvolvimento infantil de crianças de 0 a 6 anos em creches não encon- trou associação estatística entre atraso do desenvolvimento quanto ao sexo. Vale lembrar que, neste estudo a renda média das famílias foi de 7,6 salários mínimos (aproximadamente $3.800,00$ reais), enquanto que a renda médias das famílias do presente estudo foi de 968,00 reais, ou seja, muito inferior.

As outras variáveis que mostraram associação direta com suspeita de atraso no desenvolvimento neuropsicomotor no presente estudo foram: maior idade da mãe, menor Apgar no $5^{\circ}$ minuto e mães desempregadas. A questão das mães estarem desempregadas pode refletir na condição sócio-econômica da família, favorecendo um pior estímulo ao desenvolvimento das crianças. Tais associações foram diferentes com aquelas da maioria das pesquisas, onde as principais associações são: baixa renda familiar, baixa escolaridade materna e maior número de irmãos ${ }^{23,24,27}$.

Ao associar os dados do crescimento e desenvolvimento, observou-se que quanto menor o peso, o comprimento, o PC e o PT, maior o risco de atraso para o desenvolvimento neuropsicomotor. Estes achados corroboram determinada pesquisa ${ }^{28}$, que avaliou o desenvolvimento de crianças de 1 a 6 anos atendidas em uma Unidade Básica de Saúde de Porto Alegre e mostrou que o percentual de crianças com atraso no desenvolvimento foi maior no grupo com desnutrição. $\mathrm{O}$ atraso no desenvolvimento por déficit nutricional pode ser justificado devido à dificuldade de atenção durante $o$ aprendizado.

Através do exposto, observa-se que a maioria das crianças do presente estudo apresenta-se com o crescimento dentro da faixa de normalidade. Acredita-se que isto ocorra devido ao papel destes CMEIs, que tem como função social e política proporcionar à criança uma educação de qualidade, ampliando os conhecimentos físicos, culturais e sociais de forma indissociável às ações de cuidar e educar. Essas creches apresentam estrutura ampla, com agrupamentos divididos segundo a faixa etária 
e em cada agrupamento existem dois cuidadores, sendo um pedagogo e o outro auxiliar. O cardápio das crianças que frequentam estes CMEIs é orientado por uma nutricionista, proporcionando os nutrientes necessários para cada faixa etária. Neste sentido, apesar da presença de alguns fatores de risco na amostra estudada, verificou-se que a maioria das crianças apresentou crescimento normal para a sua idade. Por sua vez, a frequência de risco para problemas no desenvolvimento foi maior, considerando principalmente a área da linguagem.

Desta maneira, ao analisar o crescimento e o desenvolvimento neuropsicomotor de crianças pré-escolares de cinco CMEIs da cidade de Goiânia, pôde-se concluir que a maioria das crianças apresentou peso e comprimento dentro da faixa de normalidade. As variáveis que tiveram relação direta com o déficit do crescimento foram: maior idade materna, menor Apgar no $5^{\circ}$ minuto, crianças do sexo masculino e menor peso adquirido na gravidez pela mãe.

\section{REFERÊNCIAS}

1. BRASIL. Ministério da Saúde (BR). Agenda de compromissos para a saúde integral da criança e redução da mortalidade infantil. Brasília (DF): Ministério da Saúde/Secretaria de Políticas de Saúde; 2004.

2. Diniz RLP. O crescimento infantil e a influência de fatores intrínsecos e extrínsecos na sua evolução. Rev Pediatr. v. 8, n. 1, p. 5-7. jan./jun. 2007.

3. Sabatés AL, Mendes LCO. Perfil do crescimento e desenvolvimento de crianças entre 12 e 36 meses de idade que freqüentam uma creche municipal da cidade de Guarulhos. Cienc Cuid Saude. 2007; 6(2): 164-70.

4. Sigaud $\mathrm{CH}$. Enfermagem pediátrica. São Paulo: EPU, 1996.
Quanto ao desenvolvimento, na primeira avaliação $46 \%$ das crianças apresentaram risco para problemas de desenvolvimento, na segunda avaliação $28 \%$ de risco e na última avaliação $44 \%$, sendo que tal valor pode ter sido influenciado pelo grande risco detectado na área da linguagem. Foram encontrados valores significativos para a associação entre atraso no desenvolvimento e sexo masculino, maior idade da mãe, menor Apgar e mães desempregadas.

Pôde-se observar também, uma associação entre baixo crescimento e o maior risco de atraso para o desenvolvimento neuropsicomotor. De modo que, a alta prevalência de suspeita de atraso no desenvolvimento alerta para a questão da qualidade no cuidado com a criança.

Tais resultados possibilitam criar estratégias de prevenção de possíveis problemas no crescimento e desenvolvimento dessas crianças. Sugere-se a realização de pesquisas maiores neste âmbito, de modo a comprovar com maior precisão os resultados encontrados.

5. Biscegli TS, Polis LB, Santos LM, Vicentin M. Avaliação do estado nutricional e do desenvolvimento neuropsicomotor em crianças frequentadoras de creche. Rev Paul Pediatr. 2007; 25(4): 337-42.

6. IBGE Síntese dos indicadores sociais 2007 v.21. [acesso em: 15 mar 2010]. Disponível em: http://www.ibge.gov.br/home/ presidencia/noticias/noticia_visualiza.php?id_noticia $=987$.

7. BRASIL - Ministério da Educação. Diretrizes e bases da educação nacional. Lei $\mathrm{n}^{\mathrm{o}}$. 9.394, 1996. [cited $2007 \mathrm{Jul} 13$ ]. Available from:

http://www.planalto.gov.br/ccivil_03/ Leis/L9394.htm.

8. Caetano MJD, Silveira CRA, Gobbi LTB. Desenvolvimento motor de pré escolares no intervalo de 13 meses. Rev. Bras. 
Cineantropom. Desempenho. Hum. 2005; 7(2): 05-13.

9. Holmes SJ, Morrow AL, Pickering LK. Child-care practices: effects of social change on the epidemiology of infectious diseases and antibiotic resistance. Epidemiol Rev. 1996; 18(1): 10-28.

10. Bezerra-Filho JG, Kerr-Pontes LRS, Barreto ML. Mortalidade infantil e contexto socioeconômico no Ceará, Brasil, no período de 1991 a 2001. Rev. Bras. Saúde Mater. Infant. 2007; 7(2): 135-42.

11. Carabolante AC, Ferriani MGC. O crescimento e desenvolvimento de crianças na faixa etária de 12 a 48 meses em creche na periferia da cidade de Ribeirão Preto SP. Rev. Eletr. Enf. 2003; 5(1):28-34. Disponível em: http://www.fen.ufg.br/revista/ revista5_1/pdf/cresci.pdf

12. Gallahue DL, Ozmun JC. Compreendendo o Desenvolvimento Motor: Bebês, Crianças, Adolescentes e Adultos. São Paulo: Phorte; 2001.

13. Marcondes E. Pediatria Básica. São Paulo: Sarvier, 2004.

14. BRASIL. Ministério da Saúde: Acompanhamento do Crescimento e Desenvolvimento Infantil. Série cadernos de Atenção Básica $n^{\circ} 11$. Série A. Normas e Manuais Técnicos. Brasília-DF; 2002.

15. Grantham MS, Cheung Y, Cueto S, Glewwe P, Richter L, Strupp B. Developmental potential in the first 5 years for children in developing countries. Lancet 2007. 369: 60-70.

16. Pilz EML, Schermann LB. Determinantes biológicos e ambientais no desenvolvimento neuropsicomotor em uma amostra de crianças de Canoas/RS. Ciênc. saúde coletiva. 2007; 12(1): 181-90.

17. ABEP (Associação Brasileira de Empresas de Pesquisa). Critério Padrão de Classificação Econômica Brasil/2008.

18. Frankenburg WK, Dodds J, Archer P, Shapiro H, Bresnick B. The Denver II: a major revision and restandasdization of the Denver Developmental Screening Test. Pediatrics. 1992; 89(9): 1-7.

19. Santos ALB, Leão LSCS. Perfil antropométrico de pré-escolares de uma creche em Duque de Caxias, Rio de Janeiro. Rev. paul. pediatr. 2008; 26(3): 218-24.

20. Simon VGN, Souza JMP, Souza SB. Aleitamento materno, alimentação complemen- tar, sobrepeso e obesidade em pré-escolares. Rev. Saúde Pública. 2009; 43(1): 60-9.

21. Rocha DS, Lamounier JA, Franceschini SCC, Capanema FD, Norton RC, Oliveira VB, Sadala PV, Campos SF, Toni FA, Oliveira LS. Fatores de risco para déficit nutricional em crianças matriculadas em creches. Rev Pediatr. 2007; 8(1): 17-26.

22. Gallo PR, Leone C, Amigo H. Tendência de crescimento de filhos de mães adolescentes. Rev. bras. crescimento desenvolv. hum. 2009; 19(2): 297-305.

23. Halpern R, Barros AJD, Matijasevich A, Santos AS, Victora CG, Barros FC. Developmental status at age 12 months according to birth weight and family income: a comparison of two Brazilian birth cohorts. Cad. Saúde Pública. 2008; 24(3): 444-50.

24. Cachapuz RF, Halpern R. A influência das variáveis ambientais no desenvolvimento da linguagem em uma amostra de crianças. Rev. da AMRIGS (Associação Médica do Rio Grande do Sul). 2006; 50(4): 292-301.

25. Souza SC, Leone C, Takano AO, Moratelli HB. Desenvolvimento de pré-escolares na educação infantil em Cuiabá, Mato Grosso, Brasil. Cad. Saúde Pública. 2008; 24(8): 1917-1926.

26. Beteli VC. Acompanhamento do desenvolvimento infantil em creches [tese de mestrado]. Ribeirão Preto (SP): Faculdade de Medicina de Ribeirão Preto, Universidade de São Paulo; 2006. 89 p.

27. Santos JN, Rates SPM, Lemos SMA, Lamounier JA. Anemia em crianças de uma creche pública e as repercussões sobre o desenvolvimento de linguagem. Rev. paul. pediatr. 2009; 27(1): 67-73.

28. Saccani S, Brizola E, Giordani AP, Bach S, Resende TL, Almeida CS. Avaliação do desenvolvimento neuropsicomotor em crianças de um bairro da periferia de Porto Alegre. Scientia Medica. 2007; 17(3): 130-37.

Recebido em: 22/nov./2010
Modificado em 20/jan./2011
Aceito em28/mar./2011 